\title{
Optimizing Smartphone Micro-usage with Smartwatch Notifications
}

\author{
Laurens Bolle \\ KU Leuven \\ Celestijnenlaan 200A \\ 3001 Heverlee - Belgium \\ laurens.bolle@student.kuleuven.be \\ Robin De Croon \\ KU Leuven \\ Celestijnenlaan 200A \\ 3001 Heverlee - Belgium \\ robin.decroon@kuleuven.be \\ Erik Duval \\ KU Leuven \\ Celestijnenlaan 200A \\ 3001 Heverlee - Belgium \\ erik.duval@kuleuven.be
}

Permission to make digital or hard copies of part or all of this work for personal or classroom use is granted without fee provided that copies are not made or distributed for profit or commercial advantage and that copies bear this notice and the full citation on the first page. Copyrights for third-party components of this work must be honored. For all other uses, contact the Owner/Author. Copyright is held by the owner/author(s). MobileHCl '15 Adjunct, August 25-28, 2015, Copenhagen, Denmark ACM 978-1-4503-3653-6/15/08.

http://dx.doi.org/10.1145/2786567.2794313

\begin{abstract}
In this paper, the influence of a smartwatch application on smartphone micro-usage, brief bursts of interaction [6], among students is investigated. Our study indicates that social applications, such as WhatsApp, SMS or e-mail are used most by students. These applications tend to generate numerous notifications, which often result in brief bursts of smartphone use, which is called micro-usage. We propose three novel ways of handling notifications on a smartwatch, in order to reduce micro-usage. The first proposal receives input through on-screen buttons, the second by tapping the edges of the device and the third by drawing gestures on the screen. An evaluation of our design shows an increase of $5 \%$ of application micro-usage and a decrease of $5 \%$ of the general smartphone micro-usage.

\section{ACM Classification Keywords}

H.5.2 [Information interfaces and presentation]: User Interfaces - interaction styles.

\section{Introduction and related work}

People often use their smartphone for short interactions, such as archiving new emails. These brief bursts of interactions are called 'micro-usage' [6] and even though micro-usage is very common [3], Starner [12] claims that "smartphones have very limited potential for
\end{abstract}




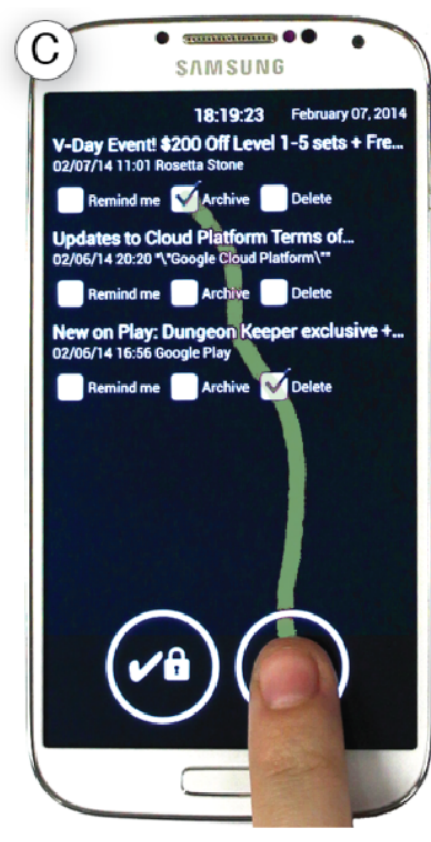

Figure 1: Interactive Android lock screen by Banovic et al. [2] which optimizes smartphone micro-usage. (Source image: [2])

Table 1: Number of sessions of the top 5 applications used in a study of 14 students for 1 week.

\begin{tabular}{ll}
\hline Application & Sessions \\
\hline Facebook & 3148 \\
Messenger & 1900 \\
SMS & 1613 \\
WhatsApp & 1453 \\
Snapchat & 953 \\
\hline
\end{tabular}

micro-interactions, which leads to users being mired and head-down in their touch screens." He believes that wearable 'wristwatch-like' interfaces will provide a maximum amount of utility for the minimum amount of user attention, helping the user to still pay attention to the real world. Today, a multitude of smartwatches are available to consumers. Typically featuring small touchscreens, they are very susceptible to the 'fat finger' problem [9]. Multiple approaches have been investigated to solve this problem on a smartwatch such as using it as a virtual peephole [7] or embedding capacitive touch sensors on the side of the watch [10].

Ferreira et al. [6] found $62 \%$ of micro-usage to be system-initiated by means of a notification. Applications most responsible for micro-usage are social applications such as Email, instant messaging and text messaging. To support this behavior of micro-usage caused by notifications, Banovic et al. [2] created an interactive Android lock screen widget, as shown in Figure 1, which allows the user to execute different actions on notifications with just one swipe, improving the mobile user experience by proactively suggesting tasks.

In summary, related research has focused on smartphone application usage and optimizing smartwatch interaction. This paper builds on this existing body of work by streamlining micro-usage for smartwatch users, while keeping the alternative input methods in mind.

\section{Student smartphone usage}

In order to understand our end-user needs, we investigated what students actually use their smartphones for. The smartphone usage of 14 students was tracked for a period of one week, by using the AWARE Framework [5]. Ferreira et al. [6] performed a similar study, with 21 participants from a general audience for a period of three weeks. They concluded that $41.5 \%$ of all application launches result in micro-usage and more than $62 \%$ of micro-sessions were caused by a notification. In our study with students, 75\% of application launches resulted in micro-usage. Social applications such as Messenger, WhatsApp or SMS generate most notifications and are used the most, as shown in Table 1. Additionally, we looked at general smartphone sessions (screen on until screen off), showing $47 \%$ of these sessions to be shorter than 15 seconds, to determine the amount of smartphone micro-usage instead of application micro-usage.

\section{Enhancing notifications}

As micro-usage is usually caused by a notification [6], our goal is to enhance smartwatch notifications to allow for better and faster handling. As Starner [12] mentions, wearable devices such as smartwatches are more suitable for micro-usage than smartphones. Since at the start of this study the Apple Watch was not yet available, we use a Samsung Gear Live smartwatch, which is based on the Android Wear platform. Android Wear already provides notifications with actions on the smartwatch, but these actions are often limited, seldom customizable, do not provide a clear overview of the possibilities and require relatively much user interaction because they are placed next to each other on different screens. The advantage of this platform is the smartwatches having a touchscreen, which operates faster than physical buttons [8]. In order to improve these notifications, we propose three ways of interacting with notifications on an Android Wear smartwatch.

The first approach divides the screen in four quadrants with a specific action, as illustrated in Figure 2. This would require only one swipe and one tap for the user to 


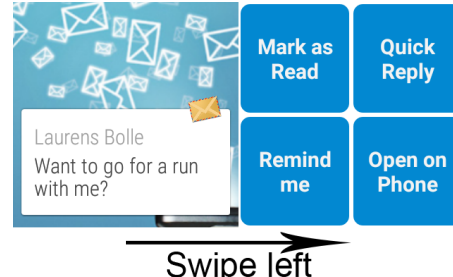

Swipe left

Figure 2: First proposal to optimise smartwatch notifications for micro-usage. After swiping the user can select one of the four (customisable) actions.

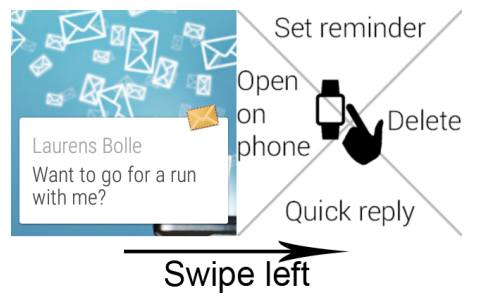

Figure 3: Second proposal: after swiping the user taps the corresponding side of the smartwatch twice to perform one of the actions.

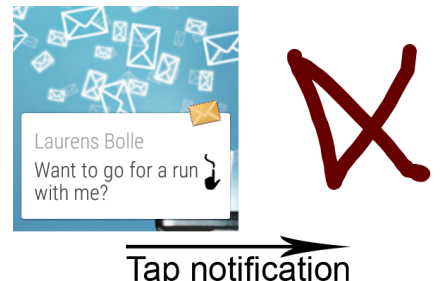

Figure 4: Third proposal: after tapping the notification, the user draws a gesture on the screen to perform an action. delete an e-mail for instance and always gives the user an overview of four possible actions. Inspired by Oakley and Lee [10], our second proposal makes use of the physical sides of the watch to give input: the user could view a notification, swipe to the left and double tap one of the sides of the device to perform an action. As illustrated by Figure 3, similar to the first proposal, the possible actions are displayed on the screen. Our third method was inspired by the Gestify application of Poppinga et al. [11] Using this method the user could draw a gesture on the screen, e.g. a large ' $X$ ' to delete an e-mail, as seen in Figure 4. This has the advantage that users could map as many actions as they would like, with the downside of having to remember the different gestures.

We tested a digital prototype of each proposal with five students (four male, one female), all 22 years old. During this evaluation, we measured which proposal is the fastest and how useful users perceived the different approaches.

To measure the speed of handling a notification, we gave the users the task to set a reminder for 2 hours of the notification using the standard way and our three proposals. We define the speed by measuring the time starting from the notification popping up on the screen, to the end of the confirmation message. Usefulness is measured with the questionnaire proposed by Davis [4]. Our results indicate the first proposal being 16\% faster than the standard way, which takes $10.43 \mathrm{~s}$ on average. The second one is $8 \%$ slower and the third one is $2 \%$ faster than the standard way. Next to being the fastest, users also perceived the first proposal to be the most useful according to the results of the questionnaire.

\section{Evaluation}

Given the results of our small usability evaluation, we made a working Android Wear application based on our first proposal, named 'WearNotifier', for our evaluation in the wild. This implementation currently supports SMS and IMAP e-mail notifications. To evaluate the implementation, eight students used WearNotifier for three to four days each. The participants are aged 22 to 24 and all are male. They started out by filling out the perceived usefulness questionnaire [4] and installing the AWARE framework [5] on their smartphone. Furthermore, in order to perform user tracking, the application creates a log entry whenever test users receive an SMS or e-mail and logs which action they use. After the test period, the students fill out the same usefulness questionnaire to see if their perception has changed about the application, followed by some open questions to situate their perceptions. Finally, the evaluation is ended with a system usability scale (SUS) questionnaire [1].

\section{Results}

Before the evaluation, the average perceived usefulness rating was 3.2 with a standard deviation of 0.5 on a scale of 1 to 5 where higher is better. After the evaluation, this increased to 3.9 with a standard deviation of 0.4 . Users indicated that the reminder function was especially more useful than they had anticipated. The SUS questionnaire resulted in an average score of 80 , meaning a 'Good' to 'Excellent' usability according to the adjective rating scale by Bangor [1], although this is only based on eight users. User tracking results indicated smartphone application micro-usage to have increased by $5 \%$, compared to our first smartphone study. One explanation would be that if a user still needs his smartphone, he does not need to spend time reading the content of the actual notification anymore, therefore increasing application micro-usage. General smartphone micro-sessions (screen on until screen off), however, decreased by $5 \%$, presumably because some notifications can be entirely handled on the smartwatch. 


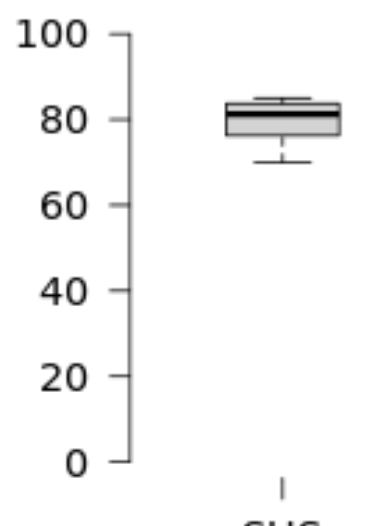

SUS

Figure 5: Boxplot of the SUS scores of the final evaluation.

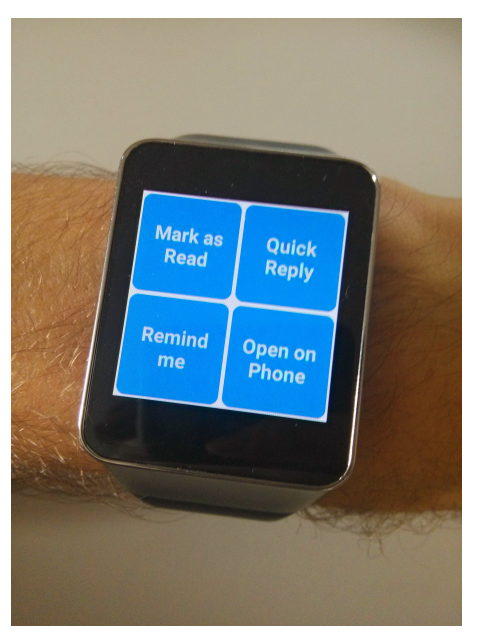

Figure 6: WearNotifier in action on the Samsung Gear Live smartwatch.

\section{Conclusion \& Future work}

Previous research [6] found notifications to be the main cause of micro-usage. We have investigated smartphone micro-usage specifically among students. Similar to the general audience of Ferreira et al. [6], social interaction applications are also the most used among students and tend to generate numerous notifications. In order to reduce this smartphone micro-usage, we designed and evaluated three proof-of-concept methods of handling notifications on a smartwatch. The preferred method was implemented and evaluated in the wild. Based on the results of this evaluation, it can be concluded that we succeeded in reducing general smartphone micro-usage by $5 \%$ with the help of a smartwatch application, but increased application micro-usage by $5 \%$ in the process as well. As this was only a preliminarily study, some limitations apply: 1) only SMS and IMAP messages are supported; 2) only one tasks is given to the users to determine the fastest method; 3 ) no qualitative feedback is collected in order to confirm why application micro-usage actually increased; 4) no error rates were recorded while determining the fastest method; 5) the influence of using only a smartwatch without WearNotifier is not tested. Future research should address these limitations and should also include a more elaborate study with more users to determine the method of preference and investigate the influence of the application on smartphone micro-usage more precisely.

\section{References}

[1] Bangor, A., Kortum, P., and Miller, J. Determining what individual sus scores mean: Adding an adjective rating scale. J. usability stud. 4, 3 (2009), 114-123.

[2] Banovic, N., Brant, C., Mankoff, J., and Dey, A ProactiveTasks: the short of mobile device use sessions. Proc. MobileHCl (2014), 243-252.
[3] Böhmer, M., Hecht, B., Schöning, J., Krüger, A., and Bauer, G. Falling asleep with angry birds, facebook and kindle: a large scale study on mobile application usage. In Proc. MobileHCl, ACM (2011), 47-56.

[4] Davis, F. D. Perceived usefulness, perceived ease of use, and user acceptance of information technology. MIS quarterly (1989), 319-340.

[5] Dey, A. K., Wac, K., Ferreira, D., Tassini, K., Hong, J.-H., and Ramos, J. Getting closer: an empirical investigation of the proximity of user to their smart phones. In Proc. Ubiquitous computing, ACM (2011), 163-172.

[6] Ferreira, D., Goncalves, J., Kostakos, V., Barkhuus, L., and Dey, A. K. Contextual experience sampling of mobile application micro-usage. Proc. MobileHCl (2014), 91-100.

[7] Kerber, F., Krüger, A., and Löchtefeld, M. Investigating the effectiveness of peephole interaction for smartwatches in a map navigation task. Proc. MobileHCl (2014), 291-294.

[8] Lee, S., and Zhai, S. The performance of touch screen soft buttons. In Proc. SIGCHI, ACM (2009), 309-318.

[9] Morgan, K. Wearable Computing: Designing a Solution to Enhance User Interactions. UW McNair Scholars Journal XIV (2014), 123-129.

[10] Oakley, I., and Lee, D. Interaction on the edge: Offset Sensing for Small Devices. In Proc. CHI (2014), 169-178.

[11] Poppinga, B., Shirazi, A. S., Henze, N., Heuten, W., and Boll, S. Understanding shortcut gestures on mobile touch devices. In Proc. MobileHCl, ACM (2014), 173-182.

[12] Starner, T. Project Glass: An Extension of the Self. IEEE Pervasive Computing 12, 2 (Apr. 2013), 14-16. 